\title{
RADIAL PROPAGATORS AND WILSON LOOPS
}

\author{
Stefan Leupold \\ Institut für Theoretische Physik, Universität Regensburg, \\ D-93040 Regensburg, Germany \\ Heribert Weigert \\ School of Physics and Astronomy, University of Minnesota, \\ Minneapolis, MN 55455, USA
}

\begin{abstract}
We present a relation which connects the propagator in the radial (Fock-Schwinger) gauge with a gauge invariant Wilson loop. It is closely related to the well-known field strength formula and can be used to calculate the radial gauge propagator. The result is shown to diverge in four-dimensional space even for free fields, its singular nature is however naturally explained using the renormalization properties of Wilson loops with cusps and self-intersections. Using this observation we provide a consistent regularization scheme to facilitate loop calculations. Finally we compare our results with previous approaches to derive a propagator in Fock-Schwinger gauge.
\end{abstract}

\footnotetext{
*stefan.leupold@physik.uni-regensburg.de
}

†weigert@mnhepw.hep.umn.edu 


\section{Introduction}

While perturbation theory for gauge fields formulated in covariant gauges is very well established [1] many aspects of non-covariant gauges are still under discussion. In principle one expects physical quantities to be independent of the chosen gauge. However this might lead to the naive conclusion that a quantum theory in an arbitrary gauge is simply obtained by inserting the respective gauge fixing term and the appropriate Faddeev-Popov ghosts in the path integral representation and reading off the Feynman rules. Unfortunately it is not so easy to obtain the correct Feynman rules, i.e. a set of rules yielding the same results for observable quantities as calculations in covariant gauges. Prominent examples are formulations in temporal and axial gauges. Such gauge choices are considered since one expects the Faddeev-Popov ghosts to decouple. However problems even start with the determination of the appropriate free gauge propagators. Temporal and axial gauge choices yield propagators plagued by gauge poles in their momentum space representation. These are caused by the fact that such gauge conditions are insufficient to completely remove the gauge degrees of freedom. The correct treatment of such poles can cause ghost fields to reappear [2], can break translational invariance [3] or both [4]. While these problems seem to be "restricted" to the evaluation of the correct gauge propagators and ghost fields, the necessity of introducing even new multi-gluon vertices appears in the Coulomb gauge [5]. These additional vertices are due to operator ordering problems which are difficult to handle in the familiar path integral approach. They give rise to anomalous interaction terms at the two-loop level [6] and cause still unsolved problems with renormalization at the three-loop level [7].

In this article we are interested in the radial (Fock-Schwinger) gauge condition

$$
x_{\mu} A^{\mu}(x)=0 .
$$

It found widespread use in the context of QCD sum-rules (e.g. [8]). There it is used as being more or less synonymous to the important field strength formula

$$
A_{\mu}^{\mathrm{rad}}(x)=\int_{0}^{1} d s s x^{\nu} F_{\nu \mu}(s x)
$$

which enormously simplifies the task of organizing the operator product expansion of QCD n-point functions in terms of gauge invariant quantities by expressing the gauge potential via the gauge covariant field strength tensor. It was introduced long ago [9, [10] and rediscovered several times (e.g. [11]).

Only a few efforts have been made to establish perturbation theory for radial gauge. The main reason for this is that the gauge condition breaks translational invariance since the origin (in general an arbitrary but fixed point $z$, c.f. (1.6)) is singled out by the gauge condition. Thus perturbation theory cannot be formulated in momentum space as usual but must be set up in coordinate space. 
The first attempt to evaluate the free radial propagator was performed in [12]. Later however the function $\Gamma_{\mu \nu}(x, y)$ presented there was shown to be not symmetric [13]. Moreover it could not be symmetrized by adding $\Gamma_{\nu \mu}(y, x)$ since the latter is not a solution of the free Dyson equation. It was even suspected in [13] that it might be impossible to find a symmetric solution of this equation in four-dimensional space, due to the appearance of divergences even on the level of the free propagator when one uses the field strength formula to derive a free propagator. Indeed we agree with this statement in principle, but we will present an explanation for this problem and a way to bypass it. Other approaches to define a radial gauge propagator try to circumvent the problem (e.g. [14 ) by sacrificing the field strength formula as given in (1.2) which was one of the main reasons the gauge became popular in non-perturbative QCD sum rule calculations [B] in the first place. If we are not prepared to do so we are forced to understand the origin of the divergences that plague most of the attempts to define even free propagators in radial gauges and see whether they can be dealt with in a satisfying manner.

In Section 2 we will make the first and decisive step in this direction by exploring the completeness of the gauge condition (1.1) and its relation to the field strength formula and developing a new representation of the gauge potentials via link operators.

In Section 3 we use this information to relate the divergences encountered in some of the attempts to define radial propagators to the renormalization properties of link operators. We find that even free propagators in radial gauge may feel remnants of the renormalization properties of closed, gauge invariant Wilson loops. Surprising as this seems to be superficially it is not impossible however if we recall that the inhomogeneous term in the gauge transformation has an explicit $1 / g$ factor in it. As a result we are able to define a regularized radial propagator using the field strength formula and established regularization procedures for link operators.

Section 1 will be devoted to demonstrate the consistency of our approach by calculating a closed Wilson loop using our propagator and relating the steps to the equivalent calculation in Feynman gauge.

In Section 5 we obtain an explicitly finite version of our propagator by completing the renormalization program developed for link operators before we summarize and compare our results to other approaches in the literature in 6 and shortly discuss the next steps in the program of establishing a new perturbative framework in radial gauges which - although the steps to be performed are quite straightforward - we will postpone for a future publication.

In the following we work in a $D$-dimensional Euclidean space. The vector potentials are given by

$$
A_{\mu}(x) \equiv A_{\mu}^{a}(x) t_{a}
$$

where $t_{a}$ denotes the generators of an $S U(N)$ group in the fundamental repre- 
sentation obeying

$$
\left[t_{a}, t_{b}\right]=i f_{a b c} t^{c}
$$

and

$$
\operatorname{tr}\left(t_{a} t_{b}\right)=\frac{1}{2} \delta_{a b} .
$$

In general the radial gauge condition with respect to $z$ reads

$$
(x-z)_{\mu} A^{\mu}(x)=0 .
$$

For simplicity we take $z=0$ after Section 2. The results nevertheless can be easily generalized to arbitrary values of $z$.

\section{The gauge condition revisited}

Before we can go ahead and tackle the problem of divergences in the radial gauge propagator we have to establish a clearer picture of the uniqueness of the gauge condition we are about to implement. After all, if we do not succeed to fix the gauge completely we might be naturally confronted with divergences if not at the free level then later in perturbative calculations. They would be a simple consequence of the incompleteness of the gauge fixing and the zero modes of the propagator which would then necessarily be present. This point has caused a still continuing discussion for the case of axial gauges (e.g. 顿) but is only briefly mentioned in the context of radial gauges (e.g. [15]).

Readers who are not interested in the discussion of (in)completeness of radial gauge conditions might skip the following considerations without getting lost and start reading again after eq. (2.6).

To clarify the question whether the gauge condition (1.6) is sufficient to completely fix the gauge degrees of freedom we have to catalogue the gauge transformations $U[B](x)$ which transform an arbitrary vector potential $B$ into the field $A$ satisfying (1.6). A gauge condition is complete if $U[B](x)$ is uniquely determined up to a global gauge transformation. In other words, we want to find all solutions of

$$
(x-z)_{\mu} U[B](x)\left[B^{\mu}(x)-\frac{1}{i g} \partial^{\mu}\right] U[B]^{-1}(x)=0 .
$$

It is easily checked that we have an infinite family of such solutions which can all be cast in the form of a product of two gauge transformations of the form

$$
U[B](x)=V(z(x)) U[B](z(x), x) .
$$

Here

$$
U[B](z(x), x)=\mathcal{P} \exp i g \int_{x}^{z(x)} d \omega_{\mu} B^{\mu}(\omega)
$$




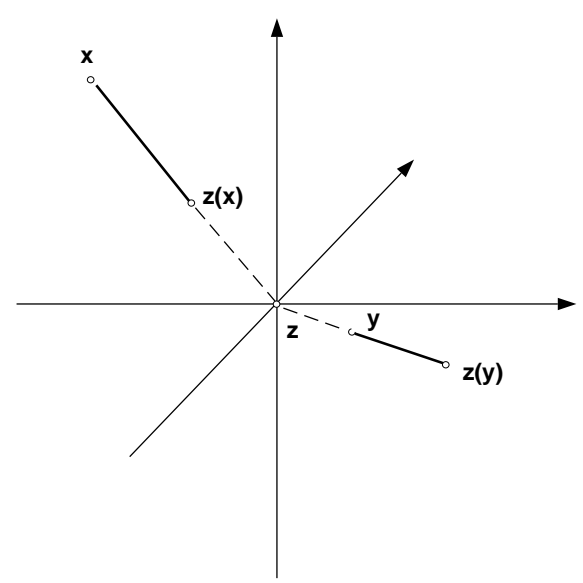

a)

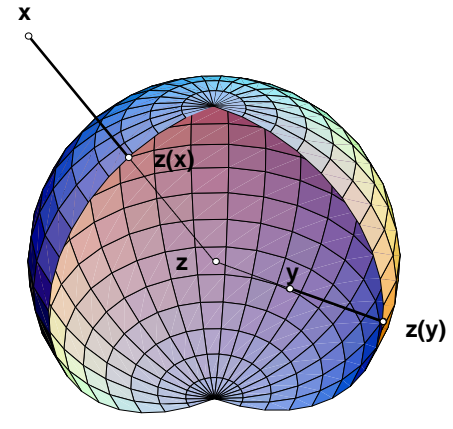

b)

Fig. 1: a) straight line path in the links for two points $x$ and $y$. b) Example for a spherical hyper-surface $z(x)$.

is a link operator whose geometric ingredients are parameterized via its endpoints $x$ and $z(x)$ and the straight line path $\omega$ between them, $\mathcal{P}$ denotes path ordering.

In particular $z(x)$ is the point where a straight line from $z$ through $x$ and a given closed hyper-surface around $z$ intersect. Since there is a unique relation between these points and the hyper-surface we will also refer to the hyper-surface itself by $z(x)$. This geometry is illustrated in Fig. 1. Both the detailed form of $z(x)$ and the local gauge transformation $V(x)$ are completely unconstrained as long as $(x-z) \cdot \partial^{x} z(x)=0$. In short, they parameterize the residual gauge freedom not eliminated by (1.1). Note that while $V(x)$ is completely arbitrary the solutions (2.2) ask only for its behavior at the given hyper-surface $z(x)$. The simplest and most intuitive choice for $z(x)$ is a spherical hyper-surface around $z$. Introducing the appropriate spherical coordinates it becomes obvious that $V(z(x))$ parameterizes gauge transformations which purely depend on the angles. Clearly the radial gauge condition (1.6) cannot fix the angular dependence of any gauge transformation in (2.1).

To eliminate the residual gauge freedom one has to impose a condition which is stronger than (1.6) and suffices to pin down $V(x)$ up to a global transformation. A possible choice for such a gauge fixing would be the condition

$$
\square\left(\int_{z(x)}^{x} d \omega \cdot A(\omega)+\int d^{4} y \frac{1}{\square}(z(x), y) \partial^{y} \cdot A(y)\right) \equiv 0
$$


which in addition to the vanishing of the radial component of the gauge potential also implements a covariant gauge on the hyper-surface $z(x)$. Such a gauge for arbitrary $z(x)$ would immediately force us to introduce ghosts into the path integral. Moreover the field strength formula would also be lost as we will illustrate below.

There is one exception to these unwanted modifications however, which may be implemented by contracting the closed surface $z(x)$ to the point $z$. Then the influence of $V(x)$ becomes degenerate with a global transformation and the gauge is completely fixed. Incidentally this is also the only case which entails the field strength formula. To see this we use

$$
\begin{aligned}
& \delta U(x, z)=i g\left\{A_{\mu}(x) U(x, z) d x^{\mu}-U(x, z) A_{\mu}(z) d z^{\mu}+\right. \\
& \left.-\int_{0}^{1} d s\left[U\left(x, w_{x}\right) F_{\mu \nu}\left(w_{x}\right) U\left(w_{x}, z\right)\right] \frac{d w_{x}^{\mu}}{d s}\left(\frac{d w_{x}^{\nu}}{d x^{\alpha}} d x^{\alpha}+\frac{d w_{x}^{\nu}}{d z^{\alpha}} d z^{\alpha}\right)\right\},
\end{aligned}
$$

(see e.g. [16], [17]) to differentiate the link operators in the gauge transformation (2.2) in order to find an expression for the radial gauge field:

$$
\begin{aligned}
A_{\mu}^{\mathrm{rad}}(x) & =U[A](z, x)\left[A_{\mu}(x)-\frac{1}{i g} \partial_{\mu}^{x}\right] U[A](x, z) \\
& =\int_{0}^{1} d s s \frac{d \omega^{\nu}}{d s} U[A](z, \omega) F_{\nu \mu}(\omega) U[A](\omega, z) \\
& =\int_{0}^{1} d s s \frac{d \omega^{\nu}}{d s} F_{\nu \mu}^{\mathrm{rad}}(\omega) .
\end{aligned}
$$

This is nothing but (1.2) for arbitrary $z$ (note that in this case $\omega=\omega(s)$ is simply given by $\omega(s)=z+(x-z) s$.) This simple result is only true since $\partial_{\mu} z(x) \equiv \partial_{\mu} z=0$. For general $z(x)$ there would be an additional term in the above formula reflecting the residual gauge freedom encoded in $V(z(x))$.

This sets the stage for a further exploration of the radial gauge in a context where we can be sure of having completely fixed the gauge in such a way that the field strength formula is guaranteed to be valid. Before we go on to studying the consequences the above has for the implementation of propagators we will introduce yet another representation of the gauge field in this particular complete radial gauge - this time solely in terms of link operators.

From now on we will assume the reference point $z$ to be the origin, but it will always be straightforward to recover the general case without any ambiguities. We will also suppress the explicit functional dependence of link operators on the gauge potential $A$ for brevity. 
Let us start with a link operator along a straight line path

$$
U\left(x, x^{\prime}\right)=\mathcal{P} \exp \left[i g \int_{0}^{1} d \omega_{\mu} A^{\mu}(\omega)\right]
$$

where now $\omega(s):=x^{\prime}+s\left(x-x^{\prime}\right)$. According to (2.5) we have

$$
\partial_{\mu}^{x} U\left(x, x^{\prime}\right)=i g\left[A_{\mu}(x)-\int_{0}^{1} d s s \frac{d \omega^{\nu}}{d s} U(x, \omega) F_{\nu \mu}(\omega) U(\omega, x)\right] U\left(x, x^{\prime}\right)
$$

which can be used to express the vector potential in terms of the link operator

$$
\lim _{x^{\prime} \rightarrow x} \partial_{\mu}^{x} U\left(x, x^{\prime}\right)=i g A_{\mu}(x) .
$$

In the case at hand the fact that $U(0, x)=1$ in any of the $x \cdot A(x)=0$ gauges allows us to introduce a new gauge covariant representation

$$
A_{\mu}^{\mathrm{rad}}(x)=\frac{1}{i g} \lim _{x^{\prime} \rightarrow x} \partial_{\mu}^{x}\left[U(0, x) U\left(x, x^{\prime}\right) U\left(x^{\prime}, 0\right)\right]
$$

for the Fock-Schwinger gauge field. It is easy to see that this is indeed equivalent to the field strength formula as given in (1.2) and consequently satisfies the same complete gauge fixing condition (i.e. (2.4) for $z(x) \rightarrow z$ ):

$$
\begin{aligned}
A_{\mu}^{\mathrm{rad}}(x) & =\frac{1}{i g} \lim _{x^{\prime} \rightarrow x} \partial_{\mu}^{x}\left[U(0, x) U\left(x, x^{\prime}\right) U\left(x^{\prime}, 0\right)\right] \\
& =\frac{1}{i g} \lim _{x^{\prime} \rightarrow x}\left[\partial_{\mu}^{x} U(0, x) U\left(x, x^{\prime}\right) U\left(x^{\prime}, 0\right)\right. \\
& \left.\quad+U(0, x) \partial_{\mu}^{x} U\left(x, x^{\prime}\right) U\left(x^{\prime}, 0\right)\right] \\
& =\frac{1}{i g} \partial_{\mu}^{x} U(0, x) U(x, 0)+U(0, x) A_{\mu}(x) U(x, 0) \\
& =\int_{0}^{1} d s s x^{\nu} F_{\nu \mu}^{\mathrm{rad}}(s x)
\end{aligned}
$$

where the last step uses (2.8), mirroring the relations in (2.6) for $z=0$.

\section{The Radial Gauge Propagator}

Having established the complete gauge fixing we are interested in, it is now straightforward to devise expressions for the propagator as a two-point function. 
According the above we know that

$$
\begin{aligned}
& \left\langle A_{\mu}(x) \otimes A_{\nu}(y)\right\rangle_{\mathrm{rad}} \\
& =\lim _{\substack{x^{\prime} \rightarrow x \\
y^{\prime} \rightarrow y}} \partial_{\mu}^{x} \partial_{\nu}^{y}\left\langle U(0, x) U\left(x, x^{\prime}\right) U\left(x^{\prime}, 0\right) \otimes U(0, y) U\left(y, y^{\prime}\right) U\left(y^{\prime}, 0\right)\right\rangle \\
& =\int_{0}^{1} d s \int_{0}^{1} d t s x^{\alpha} t y^{\beta}\left\langle U(0, s x) F_{\alpha \mu}(s x) U(s x, 0) \otimes U(0, t y) F_{\beta \nu}(t y) U(t y, 0)\right\rangle .
\end{aligned}
$$

Since we are in a fixed gauge it makes sense to perform a multiplet decomposition and for instance extract the singlet part of this propagator. The latter reduces to the free propagator in the limit $g \rightarrow 0$.

We define

$$
\operatorname{tr}\left\langle A_{\mu}(x) A_{\nu}(y)\right\rangle=\operatorname{tr}\left(t_{a} t_{b}\right) \underbrace{\left\langle A_{\mu}^{a}(x) A_{\nu}^{b}(y)\right\rangle^{\text {singlet }}}_{=: \delta^{a b} D_{\mu \nu}(x, y)}=\frac{N^{2}-1}{2} D_{\mu \nu}(x, y)
$$

to extract

$$
\begin{aligned}
& \left\langle A_{\mu}^{a}(x) A_{\nu}^{b}(y)\right\rangle_{\mathrm{rad}}^{\text {singlet }}=\delta^{a b} \frac{2}{N^{2}-1} \operatorname{tr}\left\langle A_{\mu}(x) A_{\nu}(y)\right\rangle_{\mathrm{rad}}=\delta^{a b} \frac{2}{N^{2}-1} \\
& \quad \times \frac{1}{(i g)^{2}} \lim _{\substack{x^{\prime} \rightarrow x \\
y^{\prime} \rightarrow y}} \partial_{\mu}^{x} \partial_{\nu}^{y} \operatorname{tr}\left\langle U(0, x) U\left(x, x^{\prime}\right) U\left(x^{\prime}, 0\right) U(0, y) U\left(y, y^{\prime}\right) U\left(y^{\prime}, 0\right)\right\rangle .
\end{aligned}
$$

Obviously

$$
W_{1}\left(x, x^{\prime}, y, y^{\prime}\right):=\frac{1}{N} \operatorname{tr}\left\langle U(0, x) U\left(x, x^{\prime}\right) U\left(x^{\prime}, 0\right) U(0, y) U\left(y, y^{\prime}\right) U\left(y^{\prime}, 0\right)\right\rangle
$$

is a gauge invariant Wilson loop. Its geometry is illustrated in Fig. 目.

On the other hand, using the second expression in (3.1) we have an equivalent representation for the singlet part of radial gauge propagator via the field strength formula:

$$
\begin{aligned}
& \left\langle A_{\mu}^{a}(x) A_{\nu}^{b}(y)\right\rangle_{\mathrm{rad}}^{\text {singlet }}=\delta^{a b} \frac{2}{N^{2}-1} \\
& \quad \times \int_{0}^{1} d s \int_{0}^{1} d t s x^{\alpha} t y^{\beta} \operatorname{tr}\left\langle U(0, s x) F_{\alpha \mu}(s x) U(s x, 0) U(0, t y) F_{\beta \nu}(t y) U(t y, 0)\right\rangle .
\end{aligned}
$$

Modanese [13] tried to calculate the free radial gauge propagator from (3.5) in a $D$ dimensional space-time. 5 Unfortunately one gets a result which diverges in the limit $D \rightarrow 4$.

\footnotetext{
${ }^{3}$ In fact he discussed the Abelian case but this makes no difference for free fields.
} 


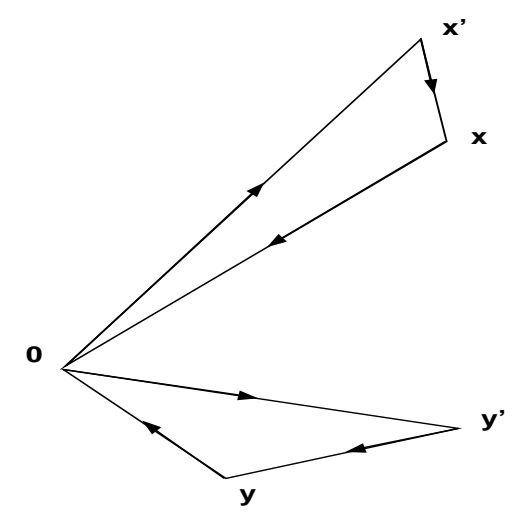

Fig. 2: The Wilson loop $W_{1}$ which is intimately connected with the radial gauge propagator according to (3.3), (3.4.))

Since we have performed a complete gauge fixing (at least on the classical level), this comes as a surprise since we certainly do not expect zero mode problems to come into the game as a possible explanation and consequently a way out. Does this mean we are trapped at a dead end or is there another explanation for this seemingly devastating discovery?

Before we try to answer this question let us briefly recapitulate how this divergence makes its appearance: Since the right hand side of (3.5) is gauge invariant we can choose an arbitrary gauge to calculate it. For simplicity we take the Feynman gauge with its free propagator

$$
\left\langle A_{\mu}^{a}(x) A_{\nu}^{b}(y)\right\rangle_{\mathrm{Feyn}}=\delta^{a b} D_{\mu \nu}^{\mathrm{Feyn}}(x, y)=-\frac{\Gamma(D / 2-1)}{4 \pi^{D / 2}} g_{\mu \nu} \delta^{a b}\left[(x-y)^{2}\right]^{1-D / 2} .
$$

Using the free field relations $U(a, b)=1$ and $F_{\mu \nu}=\partial_{\mu} A_{\nu}-\partial_{\nu} A_{\mu}$ we get (for more details see Appendix A)

$$
\begin{aligned}
\left\langle A_{\mu}^{a}(x)\right. & \left.A_{\nu}^{b}(y)\right\rangle_{\mathrm{rad}}^{0}= \\
= & -\frac{\Gamma(D / 2-1)}{4 \pi^{D / 2}} \delta^{a b} \int_{0}^{1} d s \int_{0}^{1} d t s x^{\alpha} t y^{\beta} \\
& \times\left(g_{\mu \nu} \partial_{\alpha}^{s x} \partial_{\beta}^{t y}+g_{\alpha \beta} \partial_{\mu}^{s x} \partial_{\nu}^{t y}-g_{\alpha \nu} \partial_{\mu}^{s x} \partial_{\beta}^{t y}-g_{\mu \beta} \partial_{\alpha}^{s x} \partial_{\nu}^{t y}\right)\left[(s x-t y)^{2}\right]^{1-D / 2} \\
= & -\frac{\Gamma(D / 2-1)}{4 \pi^{D / 2}} \delta^{a b}\left(g_{\mu \nu}\left[(x-y)^{2}\right]^{1-D / 2}\right. \\
& \quad-\partial_{\mu}^{x} \int_{0}^{1} d s x_{\nu}\left[(s x-y)^{2}\right]^{1-D / 2}-\partial_{\nu}^{y} \int_{0}^{1} d t y_{\mu}\left[(x-t y)^{2}\right]^{1-D / 2}
\end{aligned}
$$




$$
+\partial_{\mu}^{x} \partial_{\nu}^{y} \underbrace{\left.\int_{0}^{1} d s \int_{0}^{1} d t x \cdot y\left[(s x-t y)^{2}\right]^{1-D / 2}\right)}_{\sim \frac{1}{4-D}} .
$$

Thus the radial gauge propagator is singular for arbitrary arguments $x$ and $y$ - with one remarkable exception: It is easy to see that it vanishes for $x=0$ or $y=0$. This is simply a consequence of our task to preserve the field strength formula (1.2) which forces the vector field to vanish at the origin (in general at the reference point $z$ ).

The observation that the radial gauge propagator as calculated here diverges in four-dimensional space raises the question, whether it is perhaps impossible to formulate a quantum theory in radial gauge. This would suggest that the radial gauge condition - in the form that facilitates the field strength formula - is inherently inconsistent ("unphysical") in contrast to the general belief that it is "very physical" since it allows to express gauge variant quantities like the vector potential in terms of gauge invariant ones. To answer this question we have to understand where this divergence comes from. In the following we will see that for this purpose the complicated looking Wilson loop representation (3.3) is much more useful than the field strength formula (3.5). (Note, however, that the result for the free propagator (3.7) of course will be the same.)

It is well-known that Wilson loops need renormalization to make them welldefined (see e.g. [18] and references therein). The expansion of an arbitrary Wilson loop

$$
W(C)=\frac{1}{N} \operatorname{tr}\left\langle\mathcal{P} \exp \left[i g \oint_{C} d x^{\mu} A_{\mu}(x)\right]\right\rangle
$$

in powers of the coupling constant is given by

$$
\begin{aligned}
W(C) & =1+\frac{1}{N} \sum_{n=2}^{\infty}(i g)^{n} \oint_{C} d x_{1}^{\mu_{1}} \ldots \oint_{C} d x_{n}^{\mu_{n}} \\
& \times \Theta_{C}\left(x_{1}>\cdots>x_{n}\right) \operatorname{tr} G_{\mu_{1} \ldots \mu_{n}}\left(x_{1}, \ldots, x_{n}\right)
\end{aligned}
$$

where $\Theta_{C}\left(x_{1}>\cdots>x_{n}\right)$ orders the points $x_{1}, \ldots, x_{n}$ along the contour $C$ and

$$
G_{\mu_{1} \ldots \mu_{n}}\left(x_{1}, \ldots, x_{n}\right):=\left\langle A_{\mu_{1}}\left(x_{1}\right) \cdots A_{\mu_{n}}\left(x_{n}\right)\right\rangle
$$

are the Green functions.

In general Wilson loops show ultraviolet singularities in any order of the coupling constant. If the contour $C$ is smooth (i.e. differentiable) and simple (i.e. without self-intersections) the conventional charge and wave-function renormalization denoted by $\mathcal{R}$ in the following - is sufficient to make $W(C)$ finite. We refer to [19] for more details about renormalization of regular (smooth and simple) loops. 
In our example we must apply the renormalization operation $\mathcal{R}$ to $W_{1}$ as given in (3.4). This yields

$$
\tilde{W}_{1}\left(x, x^{\prime}, y, y^{\prime} ; g_{R}, \mu, D\right)=\mathcal{R} W_{1}\left(x, x^{\prime}, y, y^{\prime} ; g, D\right)
$$

where $W_{1}\left(x, x^{\prime}, y, y^{\prime} ; g, D\right)$ is a regularized expression calculated in $D$ dimensions and $\mu$ is a subtraction point introduced by the renormalization procedure $\mathcal{R}$. For the purpose of the present work the only important relation is

$$
g_{R}=\mu^{(D-4) / 2} g+o\left(g^{3}\right) .
$$

While the operation $\mathcal{R}$ is sufficient to make regular loops well-defined, new divergences appear if the contour $C$ has cusps or self-intersections. The renormalization properties of such loops are discussed in 20] and [21]. While the singularities of regular loops appear at the two-loop level (order $g^{4}$ in (3.9)) cusps and cross points cause divergences even in leading (non-trivial) order $g^{2}$.

Since $W_{1}$ is indeed plagued by cusps and self-intersections a second renormalization operation must be carried out to get a renormalized expression $W_{1}^{R}$ from the bare one $W_{1}$ : According to [20] each cusp is multiplicatively renormalizable with a renormalization factor $Z$ depending on the cusp angle. In our case we have four cusps with angles

$$
\begin{aligned}
\alpha & :=\angle\left(x-x^{\prime},-x\right), \\
\alpha^{\prime} & :=\angle\left(x^{\prime}, x-x^{\prime}\right), \\
\beta & :=\angle\left(y-y^{\prime},-y\right), \\
\beta^{\prime} & :=\angle\left(y^{\prime}, y-y^{\prime}\right) .
\end{aligned}
$$

The cross point at the origin introduces a mixing between $W_{1}$ and

$$
\begin{aligned}
& W_{2}\left(x, x^{\prime}, y, y^{\prime}\right):= \\
& \quad\left\langle\frac{1}{N} \operatorname{tr}\left[U(0, x) U\left(x, x^{\prime}\right) U\left(x^{\prime}, 0\right)\right] \frac{1}{N} \operatorname{tr}\left[U(0, y) U\left(y, y^{\prime}\right) U\left(y^{\prime}, 0\right)\right]\right\rangle
\end{aligned}
$$

Again the divergences appearing here are functions of the angles

$$
\left.\begin{array}{rl}
\gamma_{x x^{\prime}} & :=\angle\left(-x, x^{\prime}\right) \\
\gamma_{y y^{\prime}} & :=\angle\left(-y, y^{\prime}\right) \\
\gamma_{x y} & :=\angle(-x,-y) \\
\gamma_{x^{\prime} y^{\prime}} & :=\angle\left(x^{\prime}, y^{\prime}\right) \\
\gamma_{x^{\prime} y} & :=\angle\left(x^{\prime},-y\right) \\
\gamma_{x y^{\prime}} & :=\angle\left(-x, y^{\prime}\right)
\end{array}\right\} \vec{\gamma} .
$$

The renormalized Wilson loop $W_{1}^{R}$ is given by

$$
\begin{aligned}
& W_{1}^{R}\left(x, x^{\prime}, y, y^{\prime} ; g_{R}, \mu, \bar{C}_{\alpha}, \bar{C}_{\alpha^{\prime}}, \bar{C}_{\beta}, \bar{C}_{\beta^{\prime}}, \bar{C}_{\vec{\gamma}}\right) \\
& \quad=\lim _{D \rightarrow 4} Z\left(\bar{C}_{\alpha}, g_{R}, \mu ; D\right) Z\left(\bar{C}_{\alpha^{\prime}}, g_{R}, \mu ; D\right) Z\left(\bar{C}_{\beta}, g_{R}, \mu ; D\right) Z\left(\bar{C}_{\beta^{\prime}}, g_{R}, \mu ; D\right)
\end{aligned}
$$




$$
\begin{gathered}
\times\left[Z_{11}\left(\bar{C}_{\vec{\gamma}}, g_{R}, \mu ; D\right) \tilde{W}_{1}\left(x, x^{\prime}, y, y^{\prime} ; g_{R}, \mu, D\right)\right. \\
\left.+Z_{12}\left(\bar{C}_{\vec{\gamma}}, g_{R}, \mu ; D\right) \tilde{W}_{2}\left(x, x^{\prime}, y, y^{\prime} ; g_{R}, \mu, D\right)\right] \\
=: \lim _{D \rightarrow 4} \bar{W}_{1}\left(x, x^{\prime}, y, y^{\prime} ; g_{R}, \mu, \bar{C}_{\alpha}, \bar{C}_{\alpha^{\prime}}, \bar{C}_{\beta}, \bar{C}_{\beta^{\prime}}, \bar{C}_{\vec{\gamma}} ; D\right)
\end{gathered}
$$

where the second renormalization procedure introduces new subtraction points $\bar{C}_{\sigma}$ (c.f. [18] and [20] for more details). Of course different renormalization procedures are possible and so the $Z$ factors are not unique. We will return to this point in Section 5 where we specify a renormalization operation which is appropriate for our purposes.

The observation that Wilson loops with cusps and/or cross points show additional divergences has an important consequence for our radial gauge propagator as given in (3.3): Even the free propagator needs renormalization! This provides a natural explanation for the fact that a naive calculation of this object yields an ultraviolet divergent result [13]. Note that the usual divergences of Wilson loops which are removed by $\mathcal{R}$, like e.g. vertex divergences, appear at $o\left(g^{4}\right)$ and thus do not contribute to the free part of the radial gauge propagator, while the cusp singularities indeed contribute since they appear at $o\left(g^{2}\right)$ and affect the free field case due to the factor $1 / g^{2}$ in (3.3).

Now we are able to answer the question whether the radial gauge is "unphysical" or "very physical". It is just its intimate relation to physical, i.e. gauge invariant, quantities which makes the gauge propagator - even the free one divergent. One might cast the answer in the following form: The propagator diverges because of - and not contrary to - the fact that the radial gauge is "very physical".

Consequently the next questions are:

- Is there any use for a divergent expression for the free propagator? Especially: Can we use it to perform (dimensionally regularized) loop calculations?

- Can one find a renormalization program which yields a finite radial gauge propagator?

In the next Section we will perform a one loop calculation of a Wilson loop using the radial gauge propagator (3.7) and compare the dimensionally regularized result with a calculation in Feynman gauge.

In Section 5 we will explicitly demonstrate that the renormalization program for link operators carries over and allows to derive a finite result for the radial propagator and contrast its properties and use to the regularized version. 


\section{Calculating a Wilson Loop in Radial Gauge}

We choose the path

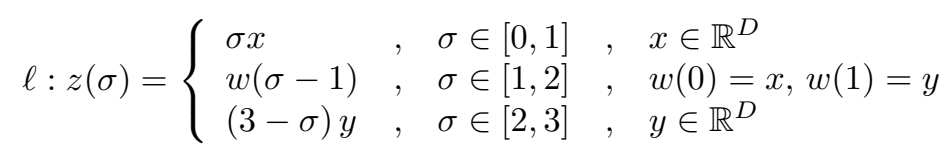

It is shown in Fig. 3. The line $w(\sigma-1)$ is supposed to be an arbitrary curve connecting $x$ and $y$.

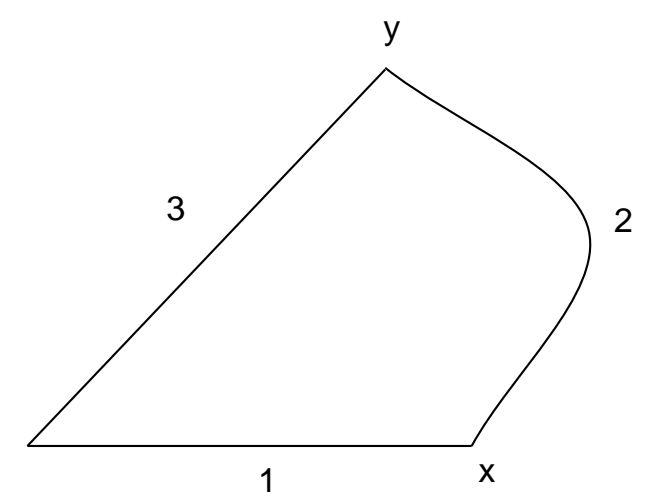

Fig. 3: A Wilson loop with two straight line parts.

First we will perform the calculation of this Wilson loop in Feynman gauge. Using (3.6) we get in leading order of the coupling constant

$$
\begin{aligned}
W(\ell) & =\frac{1}{N} \operatorname{tr}\left\langle\mathcal{P} \exp \left[i g \oint_{\ell} d z^{\mu} A_{\mu}(z)\right]\right\rangle \\
& \approx 1+(i g)^{2} \frac{N^{2}-1}{2 N} \int_{0}^{3} d \sigma \int_{0}^{3} d \tau \Theta(\sigma-\tau) \dot{z}^{\mu}(\sigma) \dot{z}^{\nu}(\tau) D_{\mu \nu}^{\mathrm{Feyn}}(z(\sigma), z(\tau)) \\
& =1+(i g)^{2} \frac{N^{2}-1}{2 N} \frac{1}{2} \underbrace{\int_{0}^{3} d \sigma \int_{0}^{3} d \tau \dot{z}^{\mu}(\sigma) \dot{z}^{\nu}(\tau) D_{\mu \nu}^{\mathrm{Feyn}}(z(\sigma), z(\tau))}_{=: I_{f}}
\end{aligned}
$$

To get rid of the $\Theta$-function we have exploited the symmetry property of twopoint Green functions

$$
D_{\mu \nu}^{\mathrm{Feyn}}(x, y)=D_{\nu \mu}^{\mathrm{Feyn}}(y, x)
$$


Decomposing the contour $\ell$ according to (4.1) we find that the Feynman propagator in (4.2) ) connects each segment of $\ell$ with itself and with all the other segments. Thus $I_{f}$ is given by

$$
I_{f}=\sum_{A=1}^{3} \sum_{B=1}^{3}(A, B)
$$

where $(A, B)$ denotes the contribution with propagators connecting loop segments $A$ and $B$ (c.f. Fig. B), e.g.

$$
\begin{aligned}
(1,2) & =\int_{0}^{1} d \sigma \int_{0}^{1} d \tau x^{\mu} \dot{w}^{\nu}(\tau) D_{\mu \nu}^{\mathrm{Feyn}}(\sigma x, w(\tau)) \\
& =-\frac{\Gamma(D / 2-1)}{4 \pi^{D / 2}} \int_{0}^{1} d \sigma \int_{0}^{1} d \tau x^{\mu} \dot{w}_{\mu}(\tau)\left[(\sigma x-w(\tau))^{2}\right]^{1-D / 2} .
\end{aligned}
$$

Next we will evaluate the same Wilson loop in radial gauge. Clearly the first and the third part of the path do not contribute if the radial gauge condition $x_{\mu} A^{\mu}(x)=0$ holds. We insert the free propagator

$$
\left\langle A_{\mu}^{a}(x) A_{\nu}^{b}(y)\right\rangle_{\mathrm{rad}}^{0}=: \delta^{a b} D_{\mu \nu}^{0}(x, y)
$$

from $(3.7)$ into

$$
\begin{aligned}
W(\ell) & =\frac{1}{N} \operatorname{tr}\left\langle\mathcal{P} \exp \left[i g \int_{0}^{1} d \sigma \dot{w}_{\mu}(\sigma) A^{\mu}(w(\sigma))\right]\right\rangle \\
& \approx 1+(i g)^{2} \frac{N^{2}-1}{2 N} \frac{1}{2} \underbrace{\int_{0}^{1} d \sigma \int_{0}^{1} d \tau \dot{w}^{\mu}(\sigma) \dot{w}^{\nu}(\tau) D_{\mu \nu}^{0}(w(\sigma), w(\tau))}_{=: I_{r}}
\end{aligned}
$$

and observe that

$$
\dot{w}_{\mu}(\sigma) \partial_{w(\sigma)}^{\mu}=\frac{d}{d \sigma} .
$$

Thus the integral in (4.7) reduces to

$$
\begin{aligned}
I_{r}=-\frac{\Gamma(D / 2-1)}{4 \pi^{D / 2}} & {\left[\int_{0}^{1} d \sigma \int_{0}^{1} d \tau \dot{w}_{\mu}(\sigma) \dot{w}^{\mu}(\tau)\left[(w(\sigma)-w(\tau))^{2}\right]^{1-D / 2}\right.} \\
& +\int_{0}^{1} d s \int_{0}^{1} d t\left(w_{\mu}(1) w^{\mu}(1)\left[(s w(1)-t w(1))^{2}\right]^{1-D / 2}\right.
\end{aligned}
$$




$$
\begin{gathered}
+w_{\mu}(0) w^{\mu}(0)\left[(s w(0)-t w(0))^{2}\right]^{1-D / 2} \\
-w_{\mu}(1) w^{\mu}(0)\left[(s w(1)-t w(0))^{2}\right]^{1-D / 2} \\
\left.-w_{\mu}(0) w^{\mu}(1)\left[(s w(0)-t w(1))^{2}\right]^{1-D / 2}\right) \\
-\int_{0}^{1} d s \int_{0}^{1} d \tau \dot{w}_{\mu}(\tau)\left(w^{\mu}(1)\left[(s w(1)-w(\tau))^{2}\right]^{1-D / 2}\right. \\
-\int_{0}^{1} d t \int_{0}^{1} d \sigma \dot{w}_{\mu}(\sigma)\left(w^{\mu}(1)\left[(w(\sigma)-t w(1))^{2}\right]^{1-D / 2}\right. \\
+(1,1)+(3,1)+(1,3)+(3,2)+(1,2)+(2,3)+(2,1) . \\
\left.\left.-w^{\mu}(0)\left[(w(\sigma)-t w(0))^{2}\right]^{1-D / 2}\right)\right]
\end{gathered}
$$

A careful analysis of (4.9) shows that it exactly coincides with the Feynman gauge calculation. This is expressed in the last line where we have denoted which parts of the loop are connected by the Feynman gauge propagator to reproduce $(4.9)$ term by term. Thus using the radial gauge propagator as given in (3.7) yields the same result as the calculation in Feynman gauge. Finally this regularized expression has to be renormalized. This can be performed without any problems according to 20]. Since we are not interested in the Wilson loop itself but in the comparison of the results obtained in radial and Feynman gauge, we will not calculate the renormalized expression for $W(\ell)$.

However a qualitative discussion of the renormalization properties of $W(\ell)$ is illuminating. By construction $W(\ell)$ has at least a cusp at the origin. (Other cusps are possible at $x$ or $y$ or along the line parameterized by $w$, but are not important for our considerations.) To give the right behavior of the Wilson loop the calculation of $W(\ell)$ in an arbitrary gauge must reproduce the cusp singularity. Usually the parameter integrals in the vicinity of the cusp do the job. For gauge choices where the propagator do not vanish in the vicinity of the origin this is automatically achieved. Let us assume for a moment that it is possible to construct a finite radial gauge propagator obeying the field strength formula (1.2) and therefore have trivial gauge factors along radial lines. Of course this is nothing but saying that there are no contributions form parts 
1 and 3 of the loop, i.e. in the vicinity of the origin. Since the propagator is assumed to be finite, there are no singular integrals corresponding to the cusp at the origin. Thus a finite radial gauge propagator cannot reproduce the correct behavior of the Wilson loop. In turn we conclude that a singular radial gauge propagator is mandatory to get the right renormalization properties of Wilson loops.

However, as we will demonstrate in the next Section, the renormalization procedure for Wilson loops can be used to devise a consistent renormalization program for the radial gauges considered here. We will apply it to write down a finite version of the free radial propagator. The generalization to higher orders in perturbation theory is straightforward. According to our considerations given above we shall show that the renormalized, thus finite version of the free radial propagator is not suitable as an input to perturbative calculations.

\section{The Renormalized Free Propagator}

We define the renormalized radial gauge propagator by

$$
\left\langle A_{\mu}^{a}(x) A_{\nu}^{b}(y)\right\rangle_{R}^{\text {singlet }}:=\lim _{D \rightarrow 4} \delta^{a b} \frac{2 N}{N^{2}-1} \frac{1}{\left(i g_{R}\right)^{2}} \mu^{D-4} \lim _{\substack{x^{\prime} \rightarrow x \\ y^{\prime} \rightarrow y}} \partial_{\mu}^{x} \partial_{\nu}^{y} \bar{W}_{1}\left(x, x^{\prime}, y, y^{\prime} ; D\right)
$$

where we have suppressed most of the other variables on which $\bar{W}_{1}$ depends (see (3.19).

From now on we will concentrate on the calculation of the renormalized free propagator $\left\langle A_{\mu}^{a}(x) A_{\nu}^{b}(y)\right\rangle_{R}^{0}$. The details of the renormalization program are presented in Appendix B. Of course the procedure is closely connected to the renormalization of cusp singularities of Wilson loops. The result is

$$
\begin{aligned}
& \left\langle A_{\mu}^{a}(x) A_{\nu}^{b}(y)\right\rangle_{R}^{0}= \\
& \quad=\lim _{D \rightarrow 4}\left(\delta^{a b} \mu^{D-4} \partial_{\mu}^{x} \partial_{\nu}^{y}\left(\frac{1}{4 \pi^{2}} \frac{1}{4-D}\left(\pi-\gamma_{x y}\right) \cot \gamma_{x y}\right)+\left\langle A_{\mu}^{a}(x) A_{\nu}^{b}(y)\right\rangle_{\mathrm{rad}}^{0}\right)
\end{aligned}
$$

Before discussing some properties of the renormalized free propagator we shall show that the counter term

$$
C_{\mu \nu}^{a b}(x, y):=\delta^{a b} \mu^{D-4} \partial_{\mu}^{x} \partial_{\nu}^{y}\left(\frac{1}{4 \pi^{2}} \frac{1}{4-D}\left(\pi-\gamma_{x y}\right) \cot \gamma_{x y}\right)
$$

exactly cancels the divergence of the propagator (3.7), i.e. that $\left\langle A_{\mu}^{a}(x) A_{\nu}^{b}(y)\right\rangle_{R}^{0}$ really is finite. To this end we use some technical results derived in Appendix C. The divergent part of the propagator (3.7) is given by

$$
U_{\mu \nu}^{a b}(x, y):=-\frac{\Gamma(D / 2-1)}{4 \pi^{D / 2}} \delta^{a b} \partial_{\mu}^{x} \partial_{\nu}^{y} \int_{0}^{1} d s \int_{0}^{1} d t x \cdot y\left[(s x-t y)^{2}\right]^{1-D / 2} .
$$


Using (C.4) and (C.14) we find

$$
\begin{aligned}
U_{\mu \nu}^{a b}(x, y) & =\frac{\Gamma(D / 2-1)}{4 \pi^{D / 2}} \delta^{a b} \partial_{\mu}^{x} \partial_{\nu}^{y} I_{2}(x,-y) \\
& =\frac{\Gamma(D / 2-1)}{4 \pi^{D / 2}} \delta^{a b} \partial_{\mu}^{x} \partial_{\nu}^{y}\left(\frac{1}{4-D}\left(\pi-\gamma_{x y}\right) \cot \left(\pi-\gamma_{x y}\right)+\text { finite }\right) \\
& =-\frac{1}{4 \pi^{2}} \delta^{a b} \partial_{\mu}^{x} \partial_{\nu}^{y}\left(\frac{1}{4-D}\left(\pi-\gamma_{x y}\right) \cot \gamma_{x y}\right)+\text { finite }
\end{aligned}
$$

and thus

$$
U_{\mu \nu}^{a b}(x, y)+C_{\mu \nu}^{a b}(x, y)=\text { finite }
$$

Note that if one tries to guess a finite expression like $\left\langle A_{\mu}^{a}(x) A_{\nu}^{b}(y)\right\rangle_{R}^{0}$ one would have to introduce a scale $\mu$ by hand without interpretation. In our derivation this scale appears naturally as the typical renormalization scale of the $\mathcal{R}$ operation.

The counter term $C_{\mu \nu}^{a b}(x, y)$ has some interesting properties. It is symmetric with respect to an exchange of all variables and it obeys the gauge condition

$$
x^{\mu} C_{\mu \nu}^{a b}(x, y)=0=C_{\mu \nu}^{a b}(x, y) y^{\nu} .
$$

Thus $\left\langle A_{\mu}^{a}(x) A_{\nu}^{b}(y)\right\rangle_{R}^{0}$ is finite in the limit $D \rightarrow 4$ but still can be interpreted as a gluonic two-point function which fulfills the radial gauge condition

$$
x^{\mu}\left\langle A_{\mu}^{a}(x) A_{\nu}^{b}(y)\right\rangle_{R}^{0}=0 .
$$

However the counter term $C_{\mu \nu}^{a b}(x, y)$ and thus also $\left\langle A_{\mu}^{a}(x) A_{\nu}^{b}(y)\right\rangle_{R}^{0}$ is ill-defined at the origin and hence conflicts with the field strength formula $(1.2)$. Note that the regularized propagator in contrast to the renormalized propagator is well defined and vanishes if one of its arguments approaches zero, as pointed out after eq. (3.7). We therefore conclude that we may use the regularized propagator in perturbative calculations and can be ensured to preserve relations like the field strength formula or eqs. (2.11) or (3.1) throughout the calculation. Although the counterterms are not well defined at the reference point itself - a property the radial gauge propagator simply inherits from renormalizing the cusp singularity of the underlying Wilson line - physical (gauge invariant) quantities are not affected, they are rendered finite and unambiguous.

\section{$6 \quad$ Summary and Outlook}

In this article we have shown how to calculate the radial gauge propagator in a $D$-dimensional space using Wilson loops. As discovered in [13] the free propagator diverges in four-dimensional space. We were able to explain this singular behavior by studying the properties of associated Wilson loops. Furthermore we 
have shown that the free propagator, in spite of being divergent in four dimensions, can be used for perturbative calculations in a (dimensionally) regularized framework and that the result for a gauge invariant quantity agrees with the calculation in Feynman gauge. Finally we have presented a renormalization procedure for the radial gauge propagator and calculated the explicit form of the renormalized free propagator. We have pointed out that any version of the radial propagator which is finite in four-dimensional space at least cannot reproduce the correct renormalization properties of Wilson loops with cusps at the reference point $z$.

It is instructive to compare the radial gauge propagators as presented here with other approaches: As discussed in Section 2 the radial gauge condition (1.1) does not completely fix the gauge degrees of freedom. Thus the field strength formula

$$
A_{\mu}(x)=\int_{0}^{1} d s s x^{\nu} F_{\nu \mu}(s x)
$$

is not the only solution of the system of equations

$$
\left\{\begin{array}{l}
x_{\mu} A^{\mu}(x)=0 \\
F_{\mu \nu}(x)=\partial_{\mu}^{x} A_{\nu}(x)-\partial_{\nu}^{x} A_{\mu}(x) .
\end{array}\right.
$$

One might add a function 13

$$
A_{\mu}^{0}(x)=\partial_{\mu}^{x} f(x)
$$

to (6.1) where $f$ is an arbitrary homogeneous function of degree 0. However any $A_{\mu}^{0}(x)$ added in in order to modify (6.1) is necessarily singular at the origin. Hence regularity at the origin may be used as a uniqueness condition [11. If we relax this boundary condition other solutions are possible, e.g.

$$
\bar{A}_{\mu}(x)=-\int_{1}^{\infty} d s s x^{\nu} F_{\nu \mu}(s x)
$$

where we must assume that the field strength vanishes at infinity. While (6.1) is the only solution which is regular at the origin, (6.4) is regular at infinity. Ignoring boundary conditions for the moment one can construct a radial gauge propagator by 14

$$
\frac{1}{2}\left(G_{\mu \nu}(x, y)+G_{\nu \mu}(y, x)\right)
$$

with

$$
G_{\mu \nu}(x, y):=-\int_{0}^{1} d s s x^{\alpha} \int_{1}^{\infty} d t t y^{\beta}\left\langle F_{\alpha \mu}(s x) F_{\beta \nu}(t y)\right\rangle .
$$

\footnotetext{
${ }^{4}$ For simplicity we discuss the QED case here. Aiming at an expression for the free gauge propagator this is no restriction of generality. For non-Abelian gauge groups c.f. [15].
} 
It turns out that this propagator is finite in four dimensions. However the price one has to pay is that boundary conditions are ignored and thus the object "lives" in the restricted space $\mathbb{R}^{4} \backslash\{0\}$ and not in $\mathbb{R}^{4}$ anymore. In our approach we insist on the field strength formula (6.1) widely used in operator product expansions [8] and on the regular behavior of vector potentials at the origin 11. One might use the propagator (6.5) to calculate the $g^{2}$-contribution to the Wilson loop on the contour (4.1). It is easy to check that the result differs from the one obtained in (4.7), (4.9). Clearly this is due to the fact that (6.5) is ill-defined at the origin.

In the above, all calculations were performed in Euclidean space. In Minkowski space Wilson loops show additional divergences if part of the contour coincides with the light cone [22]. Thus we expect the appearance of new singularities also for the radial propagator, at least if one or both of its arguments are light-like. Further investigation is required to work out the properties of the radial gauge propagator in Minkowski space.

To formulate perturbation theory in a specific gauge the knowledge of the correct free propagator is only the first step. In addition one has to check the decoupling of Faddeev-Popov ghosts in radial gauge which is suggested by the algebraic nature of the gauge condition. However the still continuing discussion about temporal and axial gauges might serve as a warning that the decoupling of ghosts for algebraic gauge conditions is far from being trivial (c.f. [2], [4] and references therein). To prove (or disprove) the decoupling of ghosts in radial gauge we expect that our Wilson loop representation of the propagator is of great advantage since it yields the possibility to calculate higher loop contributions in two distinct ways: On the one hand one might use the Wilson loop representation to calculate the full radial propagator up to an arbitrary order in the coupling constant. The appropriate Wilson loop can be calculated in any gauge, e.g. in a covariant gauge. On the other hand the radial propagator might be calculated according to Feynman rules. Since the results should coincide this might serve as a check for the validity and completeness of a set of radial gauge Feynman rules.

\section{Acknowledgments:}

HW wants to thank Alex Kovner for his invaluable patience in his role as a testing ground of new ideas. SL thanks Professor Ulrich Heinz for valuable discussions and support. During this research SL was supported in part by Deutsche Forschungsgemeinschaft and Bundesministerium für Bildung, Wissenschaft, Forschung und Technologie. HW was supported by the U.S. Department of Energy under grants No. DOE Nuclear DE-FG02-87ER-40328 and by the Alexander von Humboldt Foundation through their Feodor Lynen program. 


\section{A Derivation of the Free Radial Propagator}

The free radial propagator derived form the field strength formula shows a divergence in $D=4$, as already indicated in section 3, eq. (3.7). Here we give the details of the algebra leading to this conclusion.

The following relations summarize the steps carried out in the calculation below:

$$
\begin{gathered}
x_{\mu} \partial_{x}^{\mu}=|x| \partial_{|x|}, \\
T_{\mu \nu}(x, y):=x^{\alpha} y^{\beta}\left(g_{\mu \nu} \partial_{\alpha}^{x} \partial_{\beta}^{y}+g_{\alpha \beta} \partial_{\mu}^{x} \partial_{\nu}^{y}-g_{\alpha \nu} \partial_{\mu}^{x} \partial_{\beta}^{y}-g_{\mu \beta} \partial_{\alpha}^{x} \partial_{\nu}^{y}\right) \\
=g_{\mu \nu} \partial_{|x|} \partial_{|y|}|x||y|-\partial_{\mu}^{x} x_{\nu} \partial_{|y|}|y|-\partial_{\nu}^{y} y_{\mu} \partial_{|x|}|x|+\partial_{\mu}^{x} \partial_{\nu}^{y} x \cdot y
\end{gathered}
$$

Introducing $\hat{x}:=x /|x|$ and $u=s|x|$, we have for arbitrary $f$ :

$$
\begin{gathered}
s x_{\alpha} \partial_{\beta}^{s x} f(s x)=x_{\alpha} \partial_{\beta}^{x} f(s x), \\
\partial_{|x|} \int_{0}^{1} d s|x| f(s x)=\partial_{|x|} \int_{0}^{1} d s|x| f(s|x| \hat{x})=\partial_{|x|} \int_{0}^{|x|} d u f(u \hat{x})=f(|x| \hat{x})=f(x) .
\end{gathered}
$$

We get

$$
\begin{aligned}
-\frac{4 \pi^{D / 2}}{\Gamma(D / 2-1)}\left\langle A_{\mu}^{a}(x) A_{\nu}^{b}(y)\right\rangle_{\mathrm{rad}}^{0}= \\
=\delta^{a b} \int_{0}^{1} d s \int_{0}^{1} d t T_{\mu \nu}(s x, t y)\left[(s x-t y)^{2}\right]^{1-D / 2} \\
=\delta^{a b} T_{\mu \nu}(x, y) \int_{0}^{1} d s \int_{0}^{1} d t\left[(s x-t y)^{2}\right]^{1-D / 2} \\
=\delta^{a b}\left(g_{\mu \nu} \partial_{|x|} \partial_{|y|}|x||y|-\partial_{\mu}^{x} x_{\nu} \partial_{|y|}|y|-\partial_{\nu}^{y} y_{\mu} \partial_{|x|}|x|+\partial_{\mu}^{x} \partial_{\nu}^{y} x \cdot y\right) \\
\quad \times \int_{0}^{1} d s \int_{0}^{1} d t\left[(s x-t y)^{2}\right]^{1-D / 2} \\
=\delta^{a b}\left(g_{\mu \nu}\left[(x-y)^{2}\right]^{1-D / 2}\right. \\
\quad-\partial_{\mu}^{x} \int_{0}^{1} d s x_{\nu}\left[(s x-y)^{2}\right]^{1-D / 2}-\partial_{\nu}^{y} \int_{0}^{1} d t y_{\mu}\left[(x-t y)^{2}\right]^{1-D / 2}
\end{aligned}
$$




$$
+\partial_{\mu}^{x} \partial_{\nu}^{y} \underbrace{\int_{0}^{1} d s \int_{0}^{1} d t x \cdot y\left[(s x-t y)^{2}\right]^{1-D / 2}}_{\sim \frac{1}{4-D}}) .
$$

The divergent part of the double integral in the last line can be found in Appendix C. At the moment however the exact form of the divergence is not important.

\section{B Renormalization Program for the Free Prop- agator}

In Section 5 we discussed the effect of renormalization on the free radial propagator. Here derive in detail the appropriate renormalization procedure starting form the renormalization properties of Wilson lines. Only a few of the many possible renormalization constants will contribute to the final result.

Since in the relation between the propagator and the appropriate Wilson loop (3.3) a factor $1 / g^{2}$ is involved all quantities especially all the $Z$ 's and $W$ 's of eq. (3.19) have to be calculated up to $o\left(g_{R}^{2}\right)$. We have

$$
\begin{aligned}
\tilde{W}_{i} & =1+\left(i g_{R}\right)^{2} \delta \tilde{W}_{i}+o\left(g_{R}^{4}\right) \quad(i=1,2) \\
Z & =1+\left(i g_{R}\right)^{2} \delta Z+o\left(g_{R}^{4}\right) \\
Z_{11} & =1+\left(i g_{R}\right)^{2} \delta Z_{11}+o\left(g_{R}^{4}\right) \\
Z_{12} & =0+\left(i g_{R}\right)^{2} \delta Z_{12}+o\left(g_{R}^{4}\right)
\end{aligned}
$$

yielding

$$
\begin{aligned}
& \left\langle A_{\mu}^{a}(x) A_{\nu}^{b}(y)\right\rangle_{R}^{0}=\lim _{D \rightarrow 4} \delta^{a b} \frac{2 N}{N^{2}-1} \mu^{D-4} \\
& \quad \times \lim _{\substack{x^{\prime} \rightarrow x \\
y^{\prime} \rightarrow y}} \partial_{\mu}^{x} \partial_{\nu}^{y}\left[\begin{array}{c}
\delta Z\left(\bar{C}_{\alpha}\right)+\delta Z\left(\bar{C}_{\alpha^{\prime}}\right)+\delta Z\left(\bar{C}_{\beta}\right)+\delta Z\left(\bar{C}_{\beta^{\prime}}\right) \\
+\delta Z_{11}+\delta Z_{12}+\delta \tilde{W}_{1}
\end{array}\right] .
\end{aligned}
$$

Using the fact that up to $o\left(g_{R}^{2}\right)$ the two quantities $W_{1}$ and $\tilde{W}_{1}$ are essentially the same ${ }^{5}$ we find

$$
\begin{aligned}
& \lim _{D \rightarrow 4} \delta^{a b} \frac{2 N}{N^{2}-1} \mu^{D-4} \lim _{\substack{x^{\prime} \rightarrow x \\
y^{\prime} \rightarrow y}} \partial_{\mu}^{x} \partial_{\nu}^{y} \delta \tilde{W}_{1} \\
& \quad=\lim _{D \rightarrow 4} \delta^{a b} \frac{2 N}{N^{2}-1} \frac{1}{(i g)^{2}} \lim _{\substack{x^{\prime} \rightarrow x \\
y^{\prime} \rightarrow y}} \partial_{\mu}^{x} \partial_{\nu}^{y}\left(1+(i g)^{2} \delta W_{1}\right)
\end{aligned}
$$

\footnotetext{
${ }^{5}$ Only a factor $\mu^{D-4}$ comes in since $g_{R}$ as given in 3.12 is dimensionless in contrast to $g$.
} 


$$
\begin{aligned}
& =\left.\lim _{D \rightarrow 4} \delta^{a b} \frac{2 N}{N^{2}-1} \frac{1}{(i g)^{2}} \lim _{\substack{x^{\prime} \rightarrow x \\
y^{\prime} \rightarrow y}} \partial_{\mu}^{x} \partial_{\nu}^{y} W_{1}\right|_{g=0} \\
& =\lim _{D \rightarrow 4}\left\langle A_{\mu}^{a}(x) A_{\nu}^{b}(y)\right\rangle_{\mathrm{rad}}^{0} .
\end{aligned}
$$

To get the $\delta Z$ 's we must calculate $\delta \tilde{W}_{1}$ which is straightforward using (3.4) and (3.11). We only need the Feynman propagator (3.6) to get

$$
\begin{aligned}
\delta \tilde{W}_{1} & =-\mu^{4-D} \frac{N^{2}-1}{2 N} \frac{\Gamma(D / 2-1)}{4 \pi^{D / 2}} \\
& {\left[\left(\left|x^{\prime}\right|^{4-D}+\left|x-x^{\prime}\right|^{4-D}+|x|^{4-D}+\left|y^{\prime}\right|^{4-D}+\left|y-y^{\prime}\right|^{4-D}+|y|^{4-D}\right) I_{1}\right.} \\
& +I_{2}\left(x^{\prime}, x-x^{\prime}\right)+I_{2}\left(x-x^{\prime},-x\right)+I_{2}\left(-x, x^{\prime}\right) \\
& +I_{2}\left(y^{\prime}, y-y^{\prime}\right)+I_{2}\left(y-y^{\prime},-y\right)+I_{2}\left(-y, y^{\prime}\right) \\
& -I_{2}\left(x^{\prime},-y^{\prime}\right)+I_{2}\left(x^{\prime},-y\right)+I_{2}\left(y^{\prime},-x\right)-I_{2}(x,-y) \\
& -I_{3}\left(y^{\prime},-x^{\prime}, y-y^{\prime}\right)+I_{3}\left(y^{\prime},-x, y-y^{\prime}\right)-I_{3}\left(x^{\prime}-y^{\prime}, x-x^{\prime}, y^{\prime}-y\right) \\
& \left.-I_{3}\left(x^{\prime},-y^{\prime}, x-x^{\prime}\right)+I_{3}\left(x^{\prime},-y, x-x^{\prime}\right)\right]
\end{aligned}
$$

with

$$
\begin{aligned}
& I_{1}:=\int_{0}^{1} d s \int_{0}^{1} d t \Theta(s-t) \frac{1}{\left[(s-t)^{2}\right]^{D / 2-1}} \\
& I_{2}(p, q):=\int_{0}^{1} d s \int_{0}^{1} d t \frac{p \cdot q}{\left[(s p+t q)^{2}\right]^{D / 2-1}}
\end{aligned}
$$

and

$$
I_{3}(m, p, q):=\int_{0}^{1} d s \int_{0}^{1} d t \frac{p \cdot q}{\left[(m+s p+t q)^{2}\right]^{D / 2-1}} .
$$

In the following we are interested only in the divergent parts of these integrals. The integrals $I_{1}$ and $I_{2}$ are calculated in Appendix $\mathrm{q}$. The results are

$$
I_{1}=-\frac{1}{4-D}+\text { finite }
$$

and

$$
I_{2}(p, q)=\frac{1}{4-D} \gamma \cot \gamma+\text { finite }
$$

where $\gamma$ is the angle between $p$ and $q$. The integral $I_{3}$ is finite as long as $m \neq 0$.

To specify the renormalization factors $Z$ we choose the minimal subtraction scheme $K_{\gamma}^{\mathrm{MS}}$ as described in [18]. In dimensional regularization all the divergences are given by sums of pole terms. We define every $Z$ factor to be given just by the respective sum. The important property of this renormalization scheme is that the $Z$ factors depend on the angles only and not on the length 
of the loop or of any part of the loop. Using (B.11 and (B.12) ) the $Z$ factors can be read off from (B.7) (c.f. 21]):

$$
\begin{aligned}
\delta Z\left(\bar{C}_{\alpha}\right) & =\frac{N^{2}-1}{2 N} \frac{1}{4 \pi^{2}} \frac{1}{4-D}(\alpha \cot \alpha-1) \\
\delta Z\left(\bar{C}_{\alpha^{\prime}}\right) & =\frac{N^{2}-1}{2 N} \frac{1}{4 \pi^{2}} \frac{1}{4-D}\left(\alpha^{\prime} \cot \alpha^{\prime}-1\right) \\
\delta Z\left(\bar{C}_{\beta}\right)= & \frac{N^{2}-1}{2 N} \frac{1}{4 \pi^{2}} \frac{1}{4-D}(\beta \cot \beta-1) \\
\delta Z\left(\bar{C}_{\beta^{\prime}}\right)= & \frac{N^{2}-1}{2 N} \frac{1}{4 \pi^{2}} \frac{1}{4-D}\left(\beta^{\prime} \cot \beta^{\prime}-1\right) \\
\delta Z_{11}= & \frac{N^{2}-1}{2 N} \frac{1}{4 \pi^{2}} \frac{1}{4-D}\left[\left(\gamma_{x^{\prime} y} \cot \gamma_{x^{\prime} y}-1\right)+\left(\gamma_{x y^{\prime}} \cot \gamma_{x y^{\prime}}-1\right)\right] \\
\delta Z_{12}= & \frac{N^{2}-1}{2 N} \frac{1}{4 \pi^{2}} \frac{1}{4-D}\left[\gamma_{x x^{\prime}} \cot \gamma_{x x^{\prime}}+\gamma_{y y^{\prime}} \cot \gamma_{y y^{\prime}}\right. \\
& \left.-\left(\pi-\gamma_{x^{\prime} y^{\prime}}\right) \cot \left(\pi-\gamma_{x^{\prime} y^{\prime}}\right)-\left(\pi-\gamma_{x y}\right) \cot \left(\pi-\gamma_{x y}\right)\right] .
\end{aligned}
$$

Now we exploit the fact that only one of the angles, namely $\gamma_{x y}$, depends on $x$ and $y$. All the other ones depend only on $x$ or $y$ separately, or on none of them. This simplifies (B.5) drastically:

$$
\begin{aligned}
& \left\langle A_{\mu}^{a}(x) A_{\nu}^{b}(y)\right\rangle_{R}^{0}=\lim _{D \rightarrow 4} \delta^{a b} \frac{2 N}{N^{2}-1} \mu^{D-4} \lim _{\substack{x^{\prime} \rightarrow x \\
y^{\prime} \rightarrow y}} \partial_{\mu}^{x} \partial_{\nu}^{y}\left(\delta Z_{12}+\delta \tilde{W}_{1}\right) \\
& \quad=\lim _{D \rightarrow 4}\left(\delta^{a b} \mu^{D-4} \partial_{\mu}^{x} \partial_{\nu}^{y}\left(\frac{1}{4 \pi^{2}} \frac{1}{4-D}\left(\pi-\gamma_{x y}\right) \cot \gamma_{x y}\right)+\left\langle A_{\mu}^{a}(x) A_{\nu}^{b}(y)\right\rangle_{\mathrm{rad}}^{0}\right)
\end{aligned}
$$

where we have used (B.6) to get the last expression.

\section{Some Important Integrals}

The integrals $I_{1}$ and $I_{2}$ played an important part in the renormalization procedure of appendix $\mathrm{B}$ and determine the divergences of the naive free radial propagator introduced in section 3. They are discussed in detail below.

To calculate

$$
I_{1}:=\int_{0}^{1} d s \int_{0}^{1} d t \Theta(s-t) \frac{1}{\left[(s-t)^{2}\right]^{D / 2-1}}
$$

we introduce the substitution

$$
g=s-t \quad, \quad h=s+t
$$


to get

$$
\begin{aligned}
I_{1} & =\frac{1}{2} \int_{0}^{1} d g \int_{g}^{2-g} d h g^{2-D}=\int_{0}^{1} d g(1-g) g^{2-D} \\
& =\frac{\Gamma(2) \Gamma(3-D)}{\Gamma(5-D)}=\frac{1}{(4-D)(3-D)} .
\end{aligned}
$$

For the calculation of

$$
I_{2}(p, q):=\int_{0}^{1} d s \int_{0}^{1} d t \frac{p \cdot q}{\left[(s p+t q)^{2}\right]^{D / 2-1}} .
$$

we have to distinguish the two cases $p \neq \alpha q$ where the only divergence that appears is for $s=t=0$ and $p=\alpha q$ with an additional divergence at $s=t \alpha$. Here we will only need the former.

As a first step it is useful to separate off the divergence at the origin by the substitution

$$
\lambda=s+t \quad, \quad x=s / \lambda .
$$

This yields

$$
\begin{aligned}
I_{2}(p, q)= & \left(\int_{0}^{1 / 2} d x \int_{0}^{1 /(1-x)} d \lambda+\int_{1 / 2}^{1} d x \int_{0}^{1 / x} d \lambda\right) \lambda^{3-D} \frac{p \cdot q}{\left[(x p+(1-x) q)^{2}\right]^{D / 2-1}} \\
= & \int_{0}^{1 / 2} d x \frac{(1-x)^{D-4}}{4-D} \frac{p \cdot q}{\left[(x p+(1-x) q)^{2}\right]^{D / 2-1}} \\
& +\int_{1 / 2}^{1} d x \frac{x^{D-4}}{4-D} \frac{p \cdot q}{\left[(x p+(1-x) q)^{2}\right]^{D / 2-1}}
\end{aligned}
$$

As long as $p \neq-q$ holds there are no divergences in the $x$-integration since

$$
u(x):=x p+(1-x) q
$$

never vanishes. We introduce the angle between $p$ and $q$

$$
\cos \gamma:=\frac{p \cdot q}{|p||q|}
$$

and the substitution 18

$$
e^{2 i \psi}=\frac{x|p|+(1-x)|q| e^{i \gamma}}{x|p|+(1-x)|q| e^{-i \gamma}} .
$$




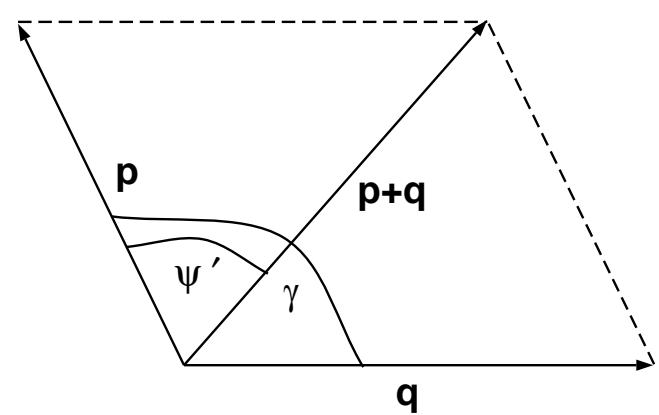

Fig. 4: The geometry of the variables appearing in the calculation of $I_{2}$ C.4).

Note that $\psi$ is nothing but the angle between $p$ and $u(x)$. To perform this substitution in (C.6) we need

$$
\begin{array}{r}
x=|q| \sin (\gamma-\psi) / N(\psi) \quad, \quad 1-x=|p| \sin \psi / N(\psi), \\
{[u(x)]^{2}=p^{2} q^{2} \sin ^{2} \gamma /[N(\psi)]^{2} \quad \text { and } \quad \frac{d \psi}{d x}=-\frac{[N(\psi)]^{2}}{|p||q| \sin \gamma}}
\end{array}
$$

with

$$
N(\psi):=|p| \sin \psi+|q| \sin (\gamma-\psi) .
$$

In addition it is useful to introduce

$$
\psi^{\prime}:=\psi(x=1 / 2)
$$

which is the angle between $p$ and $p+q$ (cf. Fig. 4).

Using all that we end up with

$$
\begin{aligned}
I_{2}(p, q) & =\int_{\gamma}^{\psi^{\prime}} d \psi \frac{-|p||q| \sin \gamma}{N^{2}}\left(\frac{|p| \sin \psi}{N}\right)^{D-4}\left(\frac{N^{2}}{p^{2} q^{2} \sin ^{2} \gamma}\right)^{D / 2-1} \frac{p \cdot q}{4-D} \\
& +\int_{\psi^{\prime}}^{0} d \psi \frac{-|p||q| \sin \gamma}{N^{2}}\left(\frac{|q| \sin (\gamma-\psi)}{N}\right)^{D-4}\left(\frac{N^{2}}{p^{2} q^{2} \sin ^{2} \gamma}\right)^{D / 2-1} \frac{p \cdot q}{4-D} \\
= & \frac{-\cos \gamma \sin ^{3-D} \gamma}{4-D}\left(|q|^{4-D} \int_{\gamma}^{\psi^{\prime}} d \psi \sin ^{D-4} \psi+|p|^{4-D} \int_{\psi^{\prime}}^{0} d \psi \sin ^{D-4}(\gamma-\psi)\right. \\
= & \frac{-\cos \gamma \sin ^{3-D} \gamma}{4-D}\left(|q|^{4-D} \int_{\gamma}^{\psi^{\prime}} d \psi \sin ^{D-4} \psi+|p|^{4-D} \int_{\gamma} d \psi \sin ^{D-4} \psi\right) \\
= & \frac{1}{4-D} \gamma \cot \gamma+\text { finite. }
\end{aligned}
$$




\section{References}

[1] P. Pascual and R. Tarrach, "QCD: Renormalization for the Practitioner", Lecture Notes in Physics, Vol. 194 (Springer, Berlin, 1984).

[2] H. Cheng and E.-C. Tsai, Phys. Rev. Lett. 57 (1986) 511.

[3] S. Caracciolo, G. Curci, and P. Menotti, Phys. Lett. B113 (1982) 311.

[4] J.-P. Leroy, J. Micheli, and G.-C. Rossi, Z. Phys. C36 (1987) 305.

[5] N.H. Christ and T.D. Lee, Phys. Rev. D22 (1980) 939.

[6] P. Doust, Ann. Phys. 177 (1987) 169.

[7] P.J. Doust and J.C. Taylor, Phys. Lett. B197 (1987) 232.

[8] M.A. Shifman, Nucl. Phys. B173 (1980) 13.

[9] V.A. Fock, Sov. Phys. 12 (1937) 404.

[10] J. Schwinger, Phys. Rev. 82 (1952) 684.

[11] C. Cronström, Phys. Lett. B90 (1980) 267.

[12] W. Kummer and J. Weiser, Z. Phys. C31 (1986) 105.

[13] G. Modanese, J. Math. Phys. 33 (1992) 1523.

[14] P. Menotti, G. Modanese, and D. Seminara, Ann. Phys. 224 (1993) 110.

[15] M. Azam, Phys. Lett. B101 (1981) 401.

[16] N.E. Bralic, Phys. Rev. D22 (1980) 3090.

[17] H.-Th. Elze, M. Gyulassy, and D. Vasak, Nucl. Phys. B276 (1986) 706.

[18] G.P. Korchemsky and A.V. Radyushkin, Nucl. Phys. B283 (1987) 342.

[19] V.S. Dotsenko and S.N. Vergeles, Nucl. Phys. B169 (1980) 527.

[20] R.A. Brandt, F. Neri, and M.-A. Sato, Phys. Rev. D24 (1981) 879.

[21] R.A. Brandt, A. Gocksch, M.-A. Sato, and F. Neri, Phys. Rev. D26 (1982) 3611.

[22] I.A. Korchemskaya and G.P. Korchemsky, Phys. Lett. B287 (1992) 169. 